\title{
MEJORAMIENTO GENÉTICO DE FRIJOL TIPO ANDINO PARA PANAMÁ: RESULTADOS DE UN PROYECTO DE PROFRIJOL. 1993-1996 1
}

\author{
Emigdio Rodríguez², Edwin Lorenzo ${ }^{3}$, Francisco González ${ }^{3}$
}

\begin{abstract}
RESUMEN
Mejoramiento genético de frijol tipo Andino para Panamá: Resultados de un proyecto de PROFRIJOL. 1993-1996. Se evaluó germoplasma criollo de frijol común de Panamá con el fin de obtener sus características morfológicas y compararlas con un grupo de líneas y variedades del Programa de mejoramiento genético de CIAT. El propósito fue encontrar germoplasma que pudiera superar las variedades criollas del área, en su grado de resistencia a la mustia hilachosa, buenos rendimientos, hábito de crecimiento y color de grano. Se utilizó el modelo de bloques completos al azar. Se seleccionaron cinco líneas promisorias por su rendimiento, niveles de resistencia a la mustia hilachosa, hábito de crecimiento, y color de grano para uso comercial. Tres líneas de frijol están en pruebas de validación en fincas de productores.
\end{abstract}

\begin{abstract}
Breeding of Andean bean type for Panama: Results of a PROFRIJOL project. 1993-1996. Native germplasm of a Panama common bean was evaluated in order to obtain its morphologic characteristics and compare them with a group of lines and varieties of CIAT's breeding program. The purpose was to find a germplasm that could surpass native varieties in terms of its resistance to Web Blight, yield, growing habits, and grain color. A randomized complete block design was used. Five promising lines for commercial use were selected based on yield, resistance to Web Blight, growth pattern, and grain color criteria. Three bean lines are undergoing validation trials in farms.
\end{abstract}

\section{INTRODUCCIÓN}

Las características morfoagronómicas de los cultivares comerciales utilizados por los agricultores de $\mathrm{Pa}$ namá, indican que las variedades preferidas son las criollas, las cuales poseen bajo potencial de rendimiento y son susceptibles a la mustia hilachosa, enfermedad fungosa producida por el hongo Thanatephorus сисиmeris (Frank), Donk.

Las pérdidas económicas ocasionadas por esta enfermedad alcanzan hasta un 90 por ciento, (Acosta,1988). En Panamá las pérdidas ocasionadas por el patógeno que causa la mustia hilachosa en el rendimiento de frijol son de hasta el 48,39 por ciento sin la aplicación de fungicidas, 14,66 por ciento con el manejo del productor y cero por ciento con la tecnología del IDIAP (Lorenzo,1995).
La mustia hilachosa se considera la enfermedad más destructiva del frijol, por la defoliación rápida y drástica que causa a las partes afectadas, provocando en la mayoría de los casos la pérdida total de la cosecha (Schwartz; Gálvez, 1979). La infección se inicia cuando por efecto de las lluvias, el suelo infestado con propágulos del hongo, en especial esclerocios y micelio llegan a los tejidos de las plantas, desarrollándose las primeras lesiones, las cuales para el caso de Panamá, con mucha frecuencia aparecen primero en las hojas primarias o en las trifoliadas más próximas al suelo. (Lorenzo et al., 1992).

Aunque no han sido identificadas líneas inmunes a la mustia hilachosa, se cuenta con cultivares moderadamente resistentes bajo intensa presión de la enfermedad (Lorenzo et al., 1992).

\footnotetext{
1 Presentado en la XLIII Reunión Anual del PCCMCA. Panamá, 1997.

2 Coordinador del Programa de frijol de Panamá.

3 Instituto de Investigación Agropecuaria de Panamá (IDIAP), Caisán, Panamá. Apdo. Postal 6-4391, El Dorado, Panamá 6APanamá. Tel. 771-2036 Fax 264-9270, E-mail: idiap@pananet.com
} 
El manejo integrado de la mustia hilachosa es el método que ha logrado mejores resultados a nivel de productor. Este sólo podrá lograrse efectivamente si se integran una serie de medidas, entre las cuales se contempla el uso de variedades tolerantes, la aplicación de productos químicos y la utilización de prácticas culturales. Estas prácticas contribuirán a un control más económico de la enfermedad y a disminuir los riesgos de daños al medio ambiente (Bonilla, et al., 1992).

Algunas prácticas recomendadas para evitar el ataque del patógeno que causa la mustia hilachosa son: el uso de coberturas vegetales para evitar el salpique de la gota de lluvia que llevaría el patógeno desde el suelo hasta la planta de frijol, utilizar una época de siembra adecuada, densidades apropiadas con buena distribución en el campo, la utilización de tutores cuando el hábito de crecimiento lo requiera, la rotación de cultivos no hospedantes (gramíneas en general), la eliminación de los residuos de cosecha y siembras con el sistema de mínima labranza. Las variedades muestran diferencias en cuanto a su reacción al ataque del agente causal de la enfermedad. Hasta el momento no se ha encontrado resistencia, aunque con tolerancia aceptable. Algunos cultivares como Porrillo 70, S-6308, Porrillo 1, Turrialba 1, Talamanca, BAT-1235, BAT 1230, BAT 1062, BAT 1444, representan tolerancia a la enfermedad (Bonilla et al., 1992).

Los genotipos de frijol común difieren en su infección de ataque de la mustia hilachosa. Los cultivares susceptibles exudan sustancias químicas que estimulan la formación de la infección en cuestión. Cultivares resistentes o tolerantes aparentemente no exudan estas sustancias químicas. Varios cultivares de frijol común han sido identificados como tolerantes a la infección causada por T. cucumeris pero no existe ningún informe de cultivares que posean un alto grado de resistencia o inmunidad (Schwartz; Gálvez, 1980).

En Panamá hay cinco variedades mejoradas procedentes del acervo Andino y que poseen hábito de crecimiento tipo I= Arbustivo determinado. Estas variedades son: Renacimiento; 80 días a la madurez fisiológica, es susceptible a la mustia hilachosa y posee un rendimiento de $1.587 \mathrm{~kg} / \mathrm{ha}$; la variedad Barriles tiene color de grano rojo, con 68 días a madurez fisiológica, es susceptible a la mustia hilachosa y posee rendimientos de $1.905 \mathrm{~kg} / \mathrm{ha}$; la variedad Primavera es de grano amarillo canario, alcanza el estado de madurez fisiológica a los 70 días, es susceptible a la mustia hilachosa y posee rendimientos de $1.723 \mathrm{~kg} / \mathrm{ha}$. El IDIAP recomienda las variedades IDIAP-R2 e IDIAP-
$\mathrm{C} 1$, tienen grano rosado y rojo moteado con crema, con 81 a 82 días a la madurez fisiológica, ambas variedades son de resistencia intermedia a la mustia hilachosa y poseen rendimientos que fluctúan entre 2.358 y 2.177 $\mathrm{kg} / \mathrm{ha}$ respectivamente. Estas variedades son de hábito de crecimiento tipo $\mathrm{I}=$ Arbustivo determinado.

Para 1997 habían tres líneas experimentales en pruebas de validación en fincas de productores. La línea I-57 sobresalió por su alta capacidad de resistir los ataques de la mustia hilachosa y buenos rendimientos. Las líneas I-47 y LRK-29 buenos rendimientos, buen nivel de resistencia a la mustia hilachosa y buen color de grano.

Los objetivos del presente trabajo fueron: evaluar el germoplasma criollo de Panamá con el fin de obtener las características morfológicas de los cultivares utilizados por el agricultor, seleccionar un grupo de líneas y variedades sobresalientes del Programa de Mejoramiento Genético del CIAT con el propósito de encontrar germoplasma sobresaliente, el cual sustituya las variedades criollas del área y brinde germoplasma con un mayor grado de resistencia a la mustia hilachosa, buenos rendimientos, buen crecimiento y excelente color de grano.

\section{MATERIALES Y MÉTODOS}

Los experimentos se establecieron en Caisán, distrito de Renacimiento, provincia de Chiriquí, Panamá. Caisán tiene una altura promedio de $800 \mathrm{msnm}$ con una temperatura y humedad relativa de $22^{\circ} \mathrm{C}$ y 80 por ciento respectivamente. Caisán está situado entre los $8^{\circ} 35^{\prime}$ latitud norte y los $82^{\circ} 40^{\prime}$ longitud oeste. Los suelos del área son suelos franco arenosos con un $\mathrm{pH}$ que fluctúa entre 5,3-6,3, la materia orgánica varia entre 9,2 y 14,5 por ciento, el fósforo entre 18,9 y 26,0 ppm y su condición puede ser considerada como buena.

La preparación de los suelos se realizó con dos pases de rastra más un pase de arado rotativo con el propósito de facilitar la infección de la enfermedad a través del salpique de la gota de lluvia.

El control de malezas se realizó con paraquat y antes de que germinara el frijol y a los 25 días después de la siembra con una pantalla protectora en la boquilla. La dosis aplicada fue de $0,4 \mathrm{~kg}$ i.a./ha. Donde existieron rebrotes de gramíneas se aplicó Fluazifob-butil en dosis de $0,31 \mathrm{~kg}$ i.a./ha. 
Se fertilizó con $100 \mathrm{~kg}$ de N y $50 \mathrm{~kg}$ de $\mathrm{P} 2 \mathrm{O} 5$ con base en $113,4 \mathrm{~kg}$ de fórmula comercial $18-46-0$ a la siembra más $181,4 \mathrm{~kg}$ de urea por hectárea, a los 25 días después de la siembra.

El control de insectos del suelo se realizó con carbofuran $10 \mathrm{~g}$ a una dosis de $2 \mathrm{~kg}$ de i.a./ha.

No se aplicaron fungicidas. Se realizó una aplicación de inóculo de la mustia hilachosa, 20 días después de la siembra, aumentando de esta manera la presión sobre el germoplasma a evaluar.

Los diseños experimentales utilizados fueron: bloques completamente al azar. También variaron el número de entradas de acuerdo al experimento en evaluación.

En los experimentos se colocaron testigos tolerantes (Talamanca) y susceptibles (Barriles) cada 10 materiales, para observar la distribución del inóculo en el campo y que ésta sirviera para facilitar el trabajo del mejorador.

Las variables medidas fueron porcentaje de severidad de la mustia hilachosa y rendimiento en $\mathrm{kg} / \mathrm{ha}$ al 14 por ciento de humedad. A los datos se les realizó un análisis de varianza y la prueba de medias de Duncan. $\mathrm{Al}$ porcentaje de severidad de la mustia hilachosa se le realizó una corrección aplicándole la siguiente fórmula en $\operatorname{sen}^{-1} \sqrt{ } \mathrm{x} / \mathrm{n} * 100$ ya que los valores porcentuales tienen una distribución binomial y no normal.

El modelo estadístico por el cual se analizaron los datos fue el siguiente:

$$
\begin{aligned}
& \text { Yij }=m+T i+b j+E i j \\
& i=1,2 \ldots t \\
& j=1,2, \ldots y \\
& \text { donde: }
\end{aligned}
$$

Yij = respuesta del tratamiento i-ésimo en el bloque j-ésimo.

$\mathrm{m}=$ media general o efecto general que es común a cada una de las unidades experimentales.

Eij= Error experimental o variable aleatoria que se le asume distribución normal e independencia con media cero y varianza constante (homogeneidad de varianza).

Al iniciar el programa de mejoramiento genético de frijol del IDIAP, el nuevo enfoque fue tratar de mejorar los cultivares que se tenían a la fecha. El primer paso fue caracterizar los cultivares criollos en las principales zonas frijoleras del país y donde se encontró que todos los materiales eran susceptibles al ataque de la mustia hilachosa mostrando porcentajes de severidad que fluctuaron entre 68 y 82 por ciento de tejido afectado. Estas variedades se caracterizaron también, por su bajo potencial de rendimiento en el sistema de labranza convencional, con promedios que variaron entre $654 \mathrm{y}$ $1.025 \mathrm{~kg} / \mathrm{ha}$. Estas fueron las principales consideraciones para iniciar un programa de mejoramiento en el cultivo de frijol, el alto grado de susceptibilidad a la mustia hilachosa que mostraban los cultivares y su bajo potencial de rendimiento (Cuadro 1).

Cuadro 1. Caracterización de los cultivares criollos cultivados en Panamá.

\begin{tabular}{lclc}
\hline Nombre & \% de sev m.h. & Reacción & Rend kg/ha \\
\hline Rosado & 82 & Susceptible & 787 \\
Chileno & 68 & Susceptible & 967 \\
Mantequilla & 72 & Susceptible & 771 \\
Calima & 70 & Susceptible & 654 \\
\hline
\end{tabular}

\section{RESULTADOS Y DISCUSIÓN}

El mejoramiento se inició por introducción y selección. Para el año de 1985 se tenía identificada la primera línea con características semejantes a la de las variedades criollas de Panamá. La variedad Renacimiento aún cuando poseía un grano más grande que la variedad chileno y era susceptible al ataque de la mustia hilachosa, mostró rendimientos superiores a los alcanzados por el criollo del productor (Cuadro 2). Es necesario destacar que esta línea fue identificada en uno de los viveros enviados por el CIAT a Panamá como PI313-700.

La institución recibió una gran presión por los productores, para liberar una variedad de grano rojo que sustituyera la variedad rosado criollo. En 1976 se recibiron de la Universidad de Cornell, E.U. poblaciones segregantes $\mathrm{F}_{2}$, en las cuales se habían realizado cruzamientos múltiples entre los progenitores Red Kote, Red Kloud, Jacob's Cattle y un cultivar mexicano de grano

Cuadro 2. Características de la variedad renacimiento y la variedad chileno. Caisán, Panamá.

\begin{tabular}{lccc}
\hline Variedad & \% de Sev. & Reacción & $\begin{array}{c}\text { Rendimiento } \\
\text { kg/ha }\end{array}$ \\
\hline Chileno & 68 & Susceptible & 967 \\
Renacimiento & 54 & Susceptible & 1.590 \\
\hline
\end{tabular}


negro. Para seleccionar plantas tolerantes a Mustia Hilachosa, se aprovechó la incidencia natural de esta enfermedad en las poblaciones y líneas segregantes.

En el Cuadro 3 se muestra el porcentaje de severidad de la mustia hilachosa y el rendimiento de las variedades Barriles y Primavera. Se observa que aún cuando dichas variedades resultaron susceptibles a la mustia hilachosa, también mostraron una alta capacidad de rendimiento.

Con base en el germoplasma enviado por CIAT y por PROFRIJOL, se logró identificar dos líneas en el IBYAN de grano rojo para clima medio, éstas fueron las líneas PVA-1076 y PVA-773 las cuales fueron liberadas en Panamá con el nombre de IDIAP-R2 e IDIAPC1 respectivamente, luego de ser evaluadas y validadas por varios años en Panamá.

En el Cuadro 4 se muestra el porcentaje de severidad de la mustia hilachosa y rendimiento en $\mathrm{kg} / \mathrm{ha}$ y el avance en cuanto a la reacción a la enfermedad en las variedades IDIAP-R2 e IDIAP-C1. Se observa el alto rendimiento logrado por estas líneas.

En el Cuadro 5 y 6 se muestran los datos de la reacción a la mustia hilachosa y del rendimiento en $\mathrm{kg} / \mathrm{ha}$ para las variedades IDIAP-R2 e IDIAP-C1, respectivamente. En comparación con la variedad Barriles en donde para un promedio de seis años la variedad IDIAP-R2 mostró un porcentaje de severidad del 22,7 porciento, mientras que la variedad IDIAP-C1 alcanzó un 32,46 porciento. Estos datos indican que aún las variedades poseen resistencia intermedia a la mustia hilachosa. El rendimiento en las dos variedades fue de $2.431,66$ y $2.185,10 \mathrm{~kg} / \mathrm{ha}$ respectivamente, lo que indica un alto potencial de rendimiento.

Se ha continuado con el mejoramiento genético, no sólo por introducción y evaluación sino también realizando cruzamientos entre las líneas sobresalientes en los diferentes viveros y las variedades Rosado criollo e IDIAP-R2, los cuales a pesar de su susceptibilidad y de resistencia intermedia a la mustia hilachosa poseen características aceptables como son color de grano y adaptación vegetativa excelente.

En el Cuadro 6, se encuentran las cruzas realizadas en Panamá, tratando de incorporar una resistencia mayor a las variedades tradicionalmente sembradas en la zona de Caisán.

En el Cuadro 7 se muestran las poblaciones $F_{2}$ de cruzamientos realizados en el CIAT para obtener de líneas con mayor grado de resistencia a la mustia

Cuadro 3. Características de las variedades barriles y primavera Caisán, Panamá.

\begin{tabular}{lccccc}
\hline & \multicolumn{2}{c}{ Rendimiento kg/ha } & & \multicolumn{2}{c}{$\begin{array}{c}\text { Mustia Hilachosa } \\
(\% \text { Sev. })\end{array}$} \\
\cline { 2 - 3 } \cline { 5 - 6 } Año & Barriles & Primavera & & Barriles & Primavera \\
\hline 1983 & $2.014,0$ & $1.572,0$ & & 70,0 & 66,0 \\
1984 & $1.191,0$ & - & & 80,0 & 72,0 \\
1985 & $2.100,0$ & - & & 80,0 & 74,0 \\
1986 & $1.650,0$ & $1.391,0$ & & 80,0 & 68,0 \\
1987 & $2.382,0$ & $2.091,0$ & & 75,0 & 58,0 \\
Promedio & $1.867,4$ & $1.685,0$ & & 77,5 & 67,6 \\
\hline
\end{tabular}

Cuadro 4. Prueba de Duncan para las variables porcentaje de severidad de la mustia hilachosa rendimiento en $\mathrm{kg} / \mathrm{ha}$ en las variedades IDIAP -r2 e IDIAP -c1.

\begin{tabular}{lcc}
\hline Tratamiento & \% de severidad & Rendimiento $\mathbf{~ g} / \mathbf{h a}$ \\
\hline IDIAP-R2 & $26,79 \mathrm{a}$ & $2.019,03 \mathrm{a}$ \\
IDIAP-C1 & $38,59 \mathrm{~b}$ & $1.823,58 \mathrm{~b}$ \\
Rosado (T.L.) & $46,79 \mathrm{c}$ & $1.222,35 \mathrm{c}$ \\
\hline
\end{tabular}

Cuadro 5. Reacción de rendimiento con $\%$ de severidad de las variedades IDIAP -r2 e IDIAP-c1 a los ataques de la Mustia Hilachosa a través de los años.

\begin{tabular}{|c|c|c|c|c|c|c|}
\hline \multirow[b]{2}{*}{ Año } & \multicolumn{3}{|c|}{$\%$ Severidad } & \multicolumn{3}{|c|}{ Rendimiento (kg./ha) } \\
\hline & IDIAP-R2 & IDIAP-C1 & Barriles & IDIAP-R2 & IDIAP-C1 & Barriles \\
\hline 1988 & 30,0 & 40,0 & 70,0 & $2.502,5$ & $2.220,1$ & $1.436,9$ \\
\hline 1989 & - & - & 80,0 & - & - & $1.589,0$ \\
\hline 1990 & 19,3 & 19,3 & 80,0 & $2.257,4$ & $2.169,8$ & $1.350,0$ \\
\hline 1991 & 40,0 & 25,0 & 80,0 & $2.338,0$ & $2.039,0$ & $1.446,0$ \\
\hline 1992 & 20,0 & 25,0 & 80,0 & $2.763,1$ & $2.717,6$ & $2.701,2$ \\
\hline 1993 & 21,5 & 38,0 & 75,0 & $2.297,3$ & $1.779,0$ & $1.883,4$ \\
\hline Promedio & 22,7 & 32,5 & 77,5 & $2.431,7$ & $2.185,1$ & $1.676,0$ \\
\hline
\end{tabular}


Cuadro 6. Cruzamientos realizados en Panamá en el año de 1996-97.

\begin{tabular}{l}
\hline Cruza \\
\hline IDIAP-R2 x BRB-166 \\
Rosado HII x BRB-166 \\
Rosado HII x FEB-183 \\
IDIAP-R2 x FEB-183 \\
(Rosado x Talamanca)*IDIAP-R2 \\
(IDIAP-R2 x Talamanca)*IDIAP-R2 \\
Rosado x FEB-184 \\
IDIAP-R2 x FEB-184 \\
Rosado x I-67 \\
IDIAP-R2 x I-67 \\
(IDIAP-R2 x DFA)*IDIAP-R2 \\
(Rosado x DFA) *IDIAP-R2
\end{tabular}

Cuadro 7. Poblaciones $\mathrm{F}_{2}$ evaluadas por su reacción a la Mustia Hilachosa. Panamá.

\begin{tabular}{|c|c|c|}
\hline & POBLACIÓN & $\begin{array}{c}\text { \% de Sev. } \\
\text { M.H. }\end{array}$ \\
\hline 1 & I-44* PVA 1076 & 30 \\
\hline 2 & I-44* PVA 773 & 30 \\
\hline 3 & I-44* Rosado & 60 \\
\hline 4 & I-44* ANT 30 & 50 \\
\hline 5 & $(\mathrm{G} 5686 *(\mathrm{CAN} 112 * \mathrm{CAL} 143)) * \mathrm{AFR}-251$ & 35 \\
\hline 6 & $($ G5686*(CAN $123 *$ CAL 143$)) *$ AFR-251 & 30 \\
\hline 7 & $($ G5686*(CAL146* CAL143))*AFR-251 & 35 \\
\hline 8 & $($ G5686*(FOT 7x CAL 143))*AFR-251 & 35 \\
\hline 9 & $(\mathrm{G} 5686 *(\mathrm{RAZ24}-1 * \mathrm{CAL} 143))$ AFR-251 & 30 \\
\hline 10 & $(\mathrm{G} 5686 *(\mathrm{AND} 1014 * \mathrm{CAL} 143)) * \mathrm{AFR}-251$ & 30 \\
\hline 11 & $(\mathrm{G} 5686 *(\mathrm{PAD} 135 * \mathrm{CAL} 143)) * \mathrm{AFR}-251$ & 25 \\
\hline
\end{tabular}

hilachosa. Sin embargo este año no fue posible la realización de selecciones individuales debido a la baja presión de enfermedad que se manifiestó en el experimento, solamente se muestra un valor de severidad para toda la población y éstas fueron cosechadas manualmente para el año de 1997 realizar selecciones individuales entre las plantas de cada población. La población con mejor comportamiento fue la (G5686* (PAD135*CAL143))*AFR251 con un total del 25 por ciento del tejido foliar afectado.

Se recibieron del CIAT 378 líneas de frijol para evaluar el ataque de la mustia hilachosa. La primera población fue la cruza de AFR-251 x BAT-1155 de donde se obtuvieron cuatro líneas, dos con buena reacción a la mustia hilachosa y dos con excelentes rendimientos (Cuadro 8). Estos materiales se pueden considerarse como segregantes transgresivos a la enfermedad y para el rendimiento obtenido.
Cuadro 8. Mejores materiales del cruzamiento de AFR251 x BAT-1155 por su reacción a la Mustia Hilachosa y por su rendimiento.

\begin{tabular}{lll}
\hline Tratamiento & $\begin{array}{c}\text { Severidad } \\
\mathbf{\%}\end{array}$ & $\begin{array}{l}\text { Rendi- } \\
\text { miento } \\
\mathbf{1 4 \%} \mathbf{~ H} .\end{array}$ \\
\hline AFR-251 x BAT1155-12-1-1-M-M-M & $25,00 \mathrm{~A}$ & $2.386,9 \mathrm{~A}$ \\
AFR-251 x BAT 1155-68-1-1-M-M-M $26,25 \mathrm{AB}$ & $2.333,5 \mathrm{AB}$ \\
AFR-251 & $35,00 \mathrm{E}$ & $2.153,1 \mathrm{D}$ \\
BAT-1155 & $60,00 \mathrm{H}$ & $1.608,4 \mathrm{~L}$ \\
\hline
\end{tabular}

Para la cruza de PVA-800 x BAT-1155 se seleccionaron cuatro materiales, dos por su reacción al ataque de la mustia hilachosa y dos por rendimiento (Cuadro 9).

La tercera población recibida correspondió al cruzamiento de PVA-800 x ICA-15399. Los materiales con mejor reacción al ataque del patógeno mostraron rendimientos superiores (Cuadro 10). Se debe destacar que los materiales sobresalientes eran segregantes transgresivos, ya que el promedio de ambos padres fue superado por las líneas seleccionadas.

El cruzamiento de AFR-251 x PVA-800 correspondió a la cuarta población evaluada. En ésta se identificaron las dos mejores líneas por su reacción a la mustia hilachosa y para su rendimiento (Cuadro 11).

Cuadro 9. Mejores materiales del cruzamiento del PVA800 x BAT-1155 por su reacción a la mustia hilachosa y por su rendimiento.

\begin{tabular}{lll}
\hline Tratamiento & $\begin{array}{c}\text { Severidad } \\
\text { \% }\end{array}$ & $\begin{array}{c}\text { Rendi- } \\
\text { miento } \\
\mathbf{1 4 \%} \mathbf{H} .\end{array}$ \\
\hline PVA-800 x BAT1155-64-1-1-M-M-M & $23,75 \mathrm{~A}$ & $1.660,20 \mathrm{~A}$ \\
PVA-800 x BAT1155-83-1-1-M-M-M & $30,00 \mathrm{AB}$ & $1.630,50 \mathrm{AB}$ \\
PVA-800 & $47,00 \mathrm{D}$ & $1.410,70$ \\
BAT-1155 & $60,00 \mathrm{I}$ & $1.308,40$ \\
\hline
\end{tabular}

Cuadro 10. Mejores materiales del cruzamiento de PVA800 x ICA-15399.

\begin{tabular}{lcc}
\hline Tratamiento & $\begin{array}{c}\text { Severidad } \\
\mathbf{\%}\end{array}$ & $\begin{array}{c}\text { Rendi- } \\
\text { mento } \\
\mathbf{1 4 \%} \mathbf{H} .\end{array}$ \\
\hline PVA-800 x ICA15399-31-1-1-M-M-M & 35,00 & $1.716,2 \mathrm{~A}$ \\
PVA-800 x ICA15399-13-1-1-M-M-M & 36,25 & $1.637,3 \mathrm{AB}$ \\
PVA-800 & 47,00 & $1.410,7$ \\
ICA-15399 & 80,00 & 597,50 \\
\hline
\end{tabular}


Cuadro 11. Mejores materiales del cruzamiento de AFR$251 \times$ PVA-800.

\begin{tabular}{lll}
\hline Tratamiento & $\begin{array}{c}\text { Severidad } \\
\text { \% }\end{array}$ & $\begin{array}{l}\text { Rendi- } \\
\text { miento } \\
\mathbf{1 4 \%} \mathbf{H} .\end{array}$ \\
\hline AFR-251 x PVA-800-65-1-M-M-M & $33,75 \mathrm{~A}$ & $2.307,1 \mathrm{~A}$ \\
AFR-251 x PVA-800-16-1-M-M-M & $33,75 \mathrm{~A}$ & $2.054,4 \mathrm{AB}$ \\
AFR-251 & $35,00 \mathrm{AB}$ & $2.153,1 \mathrm{AB}$ \\
PVA-800 & $47,00 \mathrm{DE}$ & $1.107,0 \mathrm{~F}$ \\
\hline
\end{tabular}

En el ensayo centroamericano de adaptación y rendimiento del grano rojo, se encontraron diferencias altamente significativas $(\mathrm{P}<0,001)$ para rendimiento en $\mathrm{kg}$ /ha al 14 por ciento de humedad y severidad de la mustia hilachosa. En el Cuadro 12, se pueden observar que las líneas MD30-18, MD 30-19 y MD 23-24 presentaron una reacción intermedia a la mustia hilachosa y con el rendimiento más alto se destacó la línea MD 23-24.

En la evaluación del VIDAC-grano rojo hubo diferencias altamente significativas $(\mathrm{P}<0,001)$ en el porcentaje de severidad de la mustia hilachosa y para valor agronómico.

En el Cuadro 13 se indican las nueve líneas con severidad de 20 por ciento y ocho líneas con valor agronómico de cuatro.

Se evaluó también el Vivero Internacional Caribeño (VICARIBE) donde se destacaron nueve líneas con menor porcentaje se severidad de la mustia hilachosa, pero ninguna de éstas superó al testigo resistente (Talamanca). Se observa que para el valor agronómico (VA) sobresalieron ocho líneas con valor de 4,0 (Cuadro 14).

En el Vivero Nacional de adaptación y rendimiento se observaron diferencias altamente significativas $(\mathrm{P}<0,001)$ para rendimiento, severidad y valor agronómico. Se puede observar en el Cuadro 15, que las líneas I-59, I-57 e I-44, presentaron los mejores valores agronómicos y menor porcentaje de severidad. En cuanto a rendimiento se destacaron las líneas I-59, I-57, I-46 y PVA 1097 con los rendimientos mayores.

El mejoramiento genético es una herramienta que ha permitido al programa de frijol de Panamá avanzar, aunque no con la rapidez necesaria, liberando cinco variedades comerciales. Se poseen líneas para validarse en campos de productores.
Cuadro 12. Rendimiento, severidad y valor agronómico del ECAR-grano rojo, 1996.

Tratamiento Severidad \% Tratamiento Rend. 14\% H.

\begin{tabular}{llll}
\hline MD 30-18 & 33,33 & MD 23-24 & $3.388,9$ \\
MD 30-19 & 33,33 & DOR 484 & $3.349,3$ \\
IDIAP-R2 & 28,33 & DICTA 113 & $3.023,6$ \\
MD 23-24 & 26,66 & DICTA 122 & $2.996,9$ \\
\hline
\end{tabular}

Cuadro 13. Porcentaje de severidad y valor agronómico de las líneas del VIDAC de grano rojo. Caisán 1996.

\begin{tabular}{lcc}
\hline Tratamiento & Severidad. \% & Valor Agronómico \\
\hline $9607-338$ & 20,00 & 4,00 \\
$9609-76-2$ & 20,00 & 4,00 \\
$9609-197-1$ & 20,00 & 4,00 \\
$9607-334$ & 20,00 & 4,00 \\
$9607-340$ & 20,00 & 4,00 \\
$9607-339$ & 20,00 & 4,00 \\
SRC 1-1-1-8 & 20,00 & \\
$9607-337$ & 20,00 & 4,00 \\
$9607-335$ & 20,00 & 4,00 \\
MD-30-37 & & 4,00 \\
Talamanca & 19,17 & 3,33 \\
\hline
\end{tabular}

Cuadro 14. Materiales sobresalientes en el VICARIBE 9697. Caisán, Panamá

\begin{tabular}{llccc}
\hline & Tratamiento & $\begin{array}{c}\text { Mustia } \\
\text { hilachosa }\end{array}$ & $\begin{array}{c}\text { Rendimiento } \\
\text { kg/ha }\end{array}$ & VA \\
\hline 1 & I-68 & 15,0 & $4.105,7$ & 6,0 \\
2 & $9457-43$ & 17,5 & $2.750,4$ & 6,0 \\
3 & MD-30-75 & 20,0 & $3.558,1$ & 5,5 \\
4 & I-37 & 20,0 & $1.480,6$ & 6,0 \\
5 & BAT-477 & 20.0 & $3.291,9$ & 5,0 \\
6 & 9607-15 & 22,5 & $3.929,2$ & 6,0 \\
7 & Mus PCH31-F5 & 22,5 & $3.327,8$ & 5,5 \\
8 & 9607-29 & 25,0 & $2.817,4$ & 4,5 \\
9 & I-58 & 25.0 & $3.392,0$ & 5,5 \\
10 & Mus PM-31-F5 & 27.0 & $4.544,5$ & 6,0 \\
11 & Talamanca & 10.0 & $2.541,9$ & 3,0 \\
12 & Barriles & 52,5 & $1.643,6$ & 7,0 \\
13 & IDIAP-R2 & 20,0 & $2.754,3$ & 5,0 \\
\hline
\end{tabular}


Cuadro 15. Rendimiento, severidad de la mustia hilachosa y valor agronómico (VA) de las líneas VINAR, 1996.

\begin{tabular}{lccccc}
\hline Tratamiento & Severidad \% & Tratamiento & Rendimiento (kg/ha) & Tratamiento & VA \\
\hline I-47 R & 22,50 & I-59 & $2.156,7$ & I-59 & 4,75 \\
I-38 R & 22,50 & I-57 & $2.073,7$ & I-57 & 4,50 \\
I-59 RM & 20,00 & I-46 & $2.049,3$ & I-44 & 4,50 \\
I-44 R & 17,50 & PVA-1097 & $2.048,2$ & & \\
I-57 RM & 11,250 & & & & \\
\hline
\end{tabular}

\section{LITERATURA CITADA}

ACOSTA, M. 1988. Manejo Integrado de la mustia hilachosa en el frijol común Phaseolus vulgaris L. Tesis. M.Sc. Universidad Nacional de Colombia. Bogotá, Colombia.109p.

BONILLA; BECERRA P.; ARAYA E. C.; LARA J. 1992. Importancia, síntomas y manejo de las principales enfermedades del frijol (Phaseolus vulgaris L.). Unidades de aprendizaje para la capacitación y tecnología de producción de frijol. CIAT-BID-PROFRIJOL No2. p. ?

¿¿¿LORENZO,E. y DEBE APARECER EL RESTO DE AUTORES. 19?? Determinación de pérdidas causadas por la mustia hilachosa (Thanatephorus cucumeris) en el cultivo del frijol común (Phaseolus vulgaris L.). Resultados de Investigación. Inédito. IDIAP. p. ?
LORENZO, E.; ACOSTA M.; ALFARO O.; GONZÁLES F. 1992. Resultados de proyectos de PROFRIJOL. Proyecto de mustia hilachosa. Mejoramiento Phaseolus vulgaris genético y control de la mustia hilachosa en el cultivo del frijol común L. Guatemala, Guatemala. p. ?

SCHWARTZ, H.; GALVEZ, G. 1979. Problemas de producción del frijol. Enfermedades, insectos, limitaciones edáficos y climáticos de Phaseolus vulgaris. CIAT. Cali. p. ?

SCHWARTZ F.H.; GALVEZ, G.E. .1980. Bean production problems: Disease, insect, of Phaseolus vulgaris L. Centro Internacional de Agricultura Tropical CIAT, Colombia. 424 p.

¿¿¿RODRÍGUEZ E.; LORENZO E.; GONZÁLEZ F. 1997 Manejo Integral del cultivo. Una alternativa para la producción sostenida de frijol poroto (Phaseolus vulgaris L.).Capacitación a extensionistas del MIDA. IDIAP. Panamá,. 20p. ? (falta en el texto) 